\title{
DATA ASSIMILATION METHOD IN FLOOD FORECASTING FOR RED RIVER SYSTEM USING HIGH PERFORMENT COMPUTER
}

\author{
Nguyen Thanh Don ${ }^{1, *}$, Nguyen Van Que ${ }^{2}$, Tran Quang Hung ${ }^{3}$, \\ Nguyen Hong Phong ${ }^{1}$ \\ ${ }^{1}$ Institute of Mechanics, Vietnam Academy of Science and Technology, Hanoi, Vietnam \\ ${ }^{2}$ Air Defence - Air Force Academy, Hanoi, Vietnam \\ ${ }^{3}$ Centre for Informatics and Computing, Vietnam Academy of Science and Technology, \\ Hanoi, Vietnam \\ *E-mail: ntdon@imech.ac.vn \\ Received November 19, 2014
}

\begin{abstract}
Around the world, the data assimilation framework has been reported to be of great interest for weather forecasting, oceanography modeling and for shallow water flows particularly for flood model. For flood model this method is a power full tool to identify time-independent parameters (e.g. Manning coefficients and initial conditions) and time-dependent parameters (e.g. inflow). This paper demonstrates the efficiency of the method to identify time-dependent parameter: inflow discharge with a real complex case Red River. Firstly, we briefly discuss about current methods for determining flow rate which encompasses the new technologies, then present the ability to recover flow rate of this method. For the case of very long time series, a temporal strategy with time overlapping is suggested to decrease the amount of memory required. In addition, some different aspects of data assimilation are covered from this case.
\end{abstract}

Keywords: 2D shallow water flows, DassFlow-Shalow, sensitivity analysis, variational data assimilation, identify discharge, Red river.

\section{INTRODUCTION}

Increasing pressures on our vital water resources signify that confidence in the quality of stream flow forecast, simulate and records is today, more than ever, an essential prerequisite for the sustainable management of these critical resources. Quantity of stream flow forecast, simulate and records need to ensure are stages (water level, velocity, concentration ...) and discharge. Generally, the water level is the easiest to measure and simulate, the velocity and concentration are more complicate and uncertain, and particularly the discharge is very much more difficult but is one of the quantities most 
important. This paper briefly discusses about advantages and disadvantages of mainly current methods for determining flow rate which encompasses the new technologies, and present detail the ability to recover flow rate of the variational data assimilation method.

Generally, we can't directly measure the discharge, we must calculate indirectly through other quantities. We can mention here some discharge calculation methods in operational use by engineers: discharge ratings using simple stage-discharge relations, discharge ratings using the velocity index method, discharge ratings using slope as a parameter, flow computation models for upland, branched and tidal streams, discharge ratings for miscellaneous hydraulic facilities, analysis and computation of discharge records using electronic methods, measurement discharge by conventional current meter methods, measurement discharge by acoustic and electromagnetic methods, measurement discharge by pre-calibrated measuring structure, measurement discharge by miscellaneous methods, indirect determination of peak discharge, World Meteorological Organization (WMO) [1]. Each method has its own advantages and disadvantages. Specially, we will briefly discuss two discharge calculation methods, which are commonly used in Vietnam and used to calculate discharge at Son Tay station: Discharge ratings using simple stage-discharge relations and Measurement discharge by acoustic Doppler current profilers. The identify discharge at Son Tay gauging station will be compared with official data and the result of the variational data assimilation method.

All these methods above are not efficient against the extreme high flow, flux flood, flow over bank, tide-affected flow ... except the variational data assimilation method. The variational data assimilation method based on the optimal control theory of partial differential equations offers a special powerful tool to recover flow rate of these flows above. Generally, to perform reliably operational simulations hydraulic models have to be constrained by using various observed data sets. Such models use numerous input parameters (e.g. parameterization of terrain roughness) and approximate data (e.g. topography), those parameters and data must be well measured or determined. The model calibration consists in forcing the model to provide outputs as close as possible to observed data by searching optimal values of its parameters. Previously, the main parameters to be calibrated in a hydraulic model are the roughness parameters (Manning coefficients) since these are difficult to determine a priori. In an operational context, a "hand" calibration is often done through trial tests with the use of point observations, such as recorded hydrographs at stream gauges. Furthermore, the recent improvements of mathematical tools provide more and more efficient means to calibrate numerical models. One of the methods consists in analyzing the sensitivity of the model's response to different input for parameters prioritization. Then sensitive parameters are calibrated using assimilation of observations (e.g. measurements at gauging stations, photos, satellite images). The variational data assimilation method based on the optimal control theory of partial differential equations (also called 4D-var method) offers a special powerful tool to fuse in an optimal sense measurements (observations) and the mathematical model. In river hydraulics, variational data assimilation methods have been used successfully for shallow water models.

In the world, especially in France, variational data assimilation methods have been used successfully for shallow water models (see e.g. [2-7]). In Vietnam, we started to 
research this method in March 2008, when Prof. FX Ledimet gave first lectures at Institute of Mechanics. Since then some research groups have begun research in Vietnam as that of T.D Nguyen, T.H. Tran, T.B. Dang. N. T. Don et al. have developed this approach to Red river system [8].

The objective of this paper is to assess the benefits of variational data assimilation method to recover inflow discharge of real Red river. We will compare discharge identify by data assimilation method with discharge by using stage-discharge relations, and by using Acoustic Doppler Current Profiler moving boat.

The paper is organized as follows. Section 2 briefly discuss two presents calculation methods in operational use by engineers. Section 3 gives a flood description. Section 4 recalls very briefly the full mathematical and numerical model, including the variational data assimilation process. Section 5 performs the sensitivity analysis tests in order to evaluate influence of each parameter. Section 6 shows the interest of assimilating water levels in order to calibrate the flow rate. In section 7 we present a temporal strategy with time overlapping to decrease the amount of memory required for the case of very long time series. Section 8 presents some conclusions and recommendations.

\section{COMPUTATION OF DISCHARGE}

In this chapter we briefly discuss two of many discharge calculation methods in operational use by engineers, which are used to calculate discharge at Son Tay station and commonly used in Vietnam: Discharge ratings using simple stage-discharge relations and Measurement discharge by acoustic Doppler current profilers. We have used these methods to identify the discharge at Son Tay gauging station and to compare at the same time with official data and the result of the variational data assimilation method.

\subsection{Official data}

Total inflow discharge flow into system Red River is from two reservoirs Da river (Hoa Binh), from Red river (Yen Bai) and from Lo river (Tuyen Quang), (see Figs. 12). According to the official data of National Center Hydrology Meteorology Forecast (CNHMF),from 0:00 12/08/2013 to 0:00 15/08/2013 total inflow discharge is not exceed $7200 \mathrm{~m}^{3} / \mathrm{s}$, (see Fig. 2).

Fig. 1 shows three main sources inflow discharge flow into Son Tay station. Fig. 2 shows the inflow discharge flow from each branches and total discharge flow into system (official data of NCHMF). We see that the discharge at Son Tay station at 7 o'clock of day 13/08/2013 did not exceed $7200 \mathrm{~m}^{3} / \mathrm{s}$.

Yet according to rating curve (relation Q-Z) of Hanoi Hydrology Station, NCHMF and from field measurements ADCP, discharge at Son Tay is $11000 \mathrm{~m}^{3} / \mathrm{s}, 8885 \mathrm{~m}^{3} / \mathrm{s}$, $8022 \mathrm{~m}^{3}$ /s corresponding always much higher, (see Tabs. 1-2). So we need another discharge calculation method to ensure the credibility. 


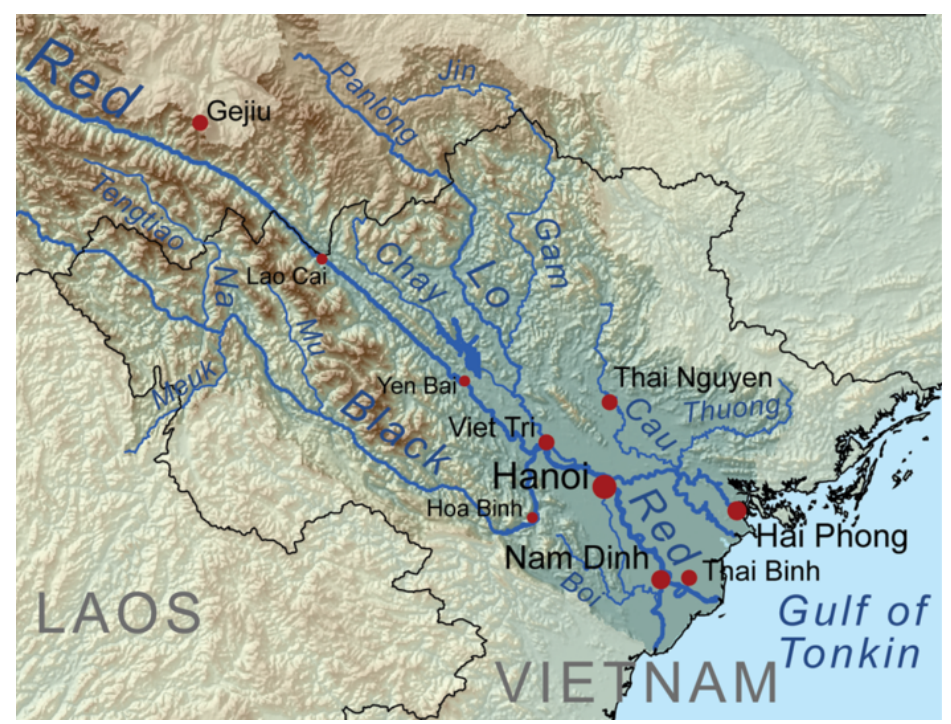

Fig. 1. Red River basin system

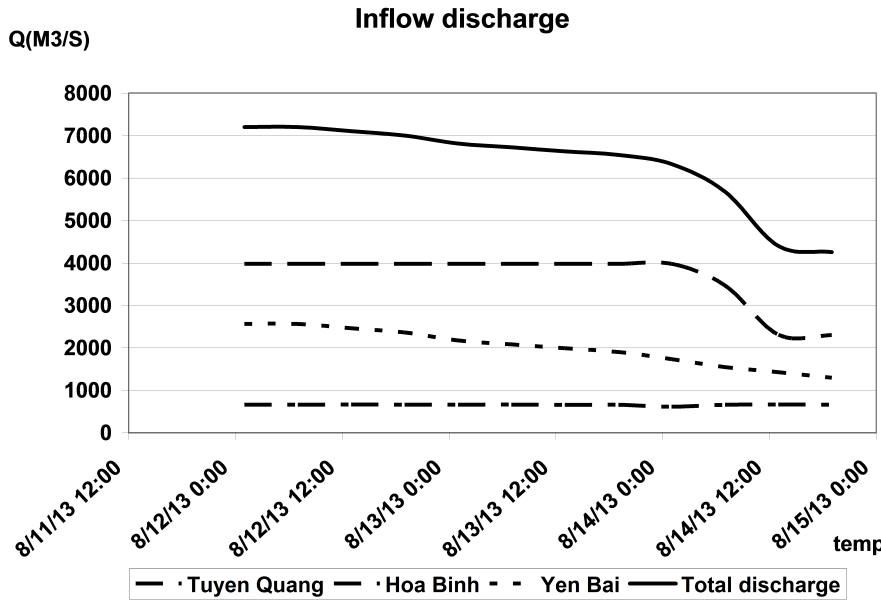

Fig. 2. Inflow discharge into system Table 1. Flow rate

\begin{tabular}{|l|c|c|}
\hline & $\begin{array}{c}\text { Discharge Son Tay } \\
\text { Station }\end{array}$ & $\begin{array}{c}\text { Percentage } \\
\text { errors }\end{array}$ \\
\hline Discharge Ratings of Hanoi Station & $11000 \mathrm{~m}^{3} / \mathrm{s}$ & $57 \%$ \\
\hline Discharge Ratings of NCHMF & $8885 \mathrm{~m}^{3} / \mathrm{s}$ & $26 \%$ \\
\hline Official data & $7000 \mathrm{~m}^{3} / \mathrm{s}$ & \\
\hline
\end{tabular}


Table 2. Flow rate

\begin{tabular}{|l|c|c|}
\hline & Discharge Son Tay Station & Percentage errors \\
\hline ADCP & $8022 \mathrm{~m}^{3} / \mathrm{s}$ & $14,6 \%$ \\
\hline Official data & $7000 \mathrm{~m}^{3} / \mathrm{s}$ & \\
\hline
\end{tabular}

\subsection{Discharge ratings using simple stage-discharge relations}

Generally, discharge rating may be complex, depending on the number of variables needed to define the stage-discharge relation. The discharge does not only depend on water level, but also on velocity and slope of free surface. However, this section is concerned with ratings in which the discharge is related to stage (water level) alone (which is used in operational use by engineers).

Discharge ratings for gauging stations are usually determined empirically by means of discharge measurements made in the field. However, it is advisable to make some current meter measurements for the purpose of confirming the pre-calibrated rating. Common practice is to measure the discharge of the stream periodically, usually by current meter, and to note the concurrent stage. At new station many discharge measurements are needed to define the stage discharge relation throughout the entire range of stage. Periodic measurements are needed thereafter to either confirm the stability of rating or to follow changes (shifts) in rating. A minimum of ten discharge measurements per year is recommended, unless it has been demonstrated that the stage-discharge relation is completely unvarying with time. In that event the frequency of measurements may be reduced (WMO [1]).

If the discharge measurements cover the entire range of stage experienced during a period of time when the stage-discharge relation is stable, there is little problem in defining the discharge rating for that period. On the other hand, if, there aren't discharge measurements to define the upper end of the rating the defined lower part of the rating curve must be extrapolated to the highest stage experienced. Such extrapolations are always subject of error, but the error may be minimized if the analyst has knowledge of the principles that govern the shape of rating curves. There are many problem of extending the high water end of rating curve, the hydrologist must be faced and decide whether the extrapolation should be straight line or whether it should be concave upward and concave downward. The problem of extrapolation can contain some uncertainty. In some case the lower end of rating curve may also need extrapolation (WMO [1]).

If we consider the discharge from official data is standard, Tab. 1 shows the inflow discharge at Son Tay gauging station and comparing performances from rating curve $Q-Z$ of Hydrology Station Hanoi, NCHMF. We found that these two results have much error, especially rating curve of Hydrology Station Hanoi.

\subsection{Measurement discharge by acoustic Doppler current profilers}

ADCP discharge measurements are becoming more and more widespread and considered am excellent and accurate means of obtaining flow data (WMO [1]). 
Nguyen Thanh Don, Nguyen Van Que, Tran Quang Hung, Nguyen Hong Phong

At site with significant backwater stable stage-discharge relations are not possible and on large streams and estuaries conventional methods of measuring discharge by current meter are impractical or very costly. Gauging sites may be inundated or inaccessible during floods. During unsteady flow conditions, measurements need to be made as rapidly as possible. Measurements on tide-affected rivers must not only be made rapidly, but often continuously, throughout a tidal cycle. Acoustic discharge measurement methods have become widespread (WMO [1]).

However, an extensive study of ADCP discharge measurement uncertainty is not yet available. Determination of uncertainty for these measurements is an extremely complex procedure. Many factor and error sources enter into any mathematical derivation of ADCP uncertainty. Work is still needed to be sure all error sources (WMO [1]).

Comparative studies of ADCP measurements with other types of measurements, such as current meter measurements, and with stage-discharge ratings, indicate that ADCP measurements provide acceptable accuracy (WMO [1]).

\section{STUDY AREA}

The computation domain is the area of Red River from Dong Anh (Thang Long bridge) to the junction of Dau branched to Duong and flowing through the Hanoi to Hung Yen reached a length of about $30 \mathrm{~km}$ (see Fig. 3). Simulation time is at 7:00 AM, 2013-08-13. Data of flow rate and water levels at many hydraulic stations from 7:00 AM to 7:00 PM were collected from various sources.

\subsection{Inflow discharge boundary}

To examine the performance of variational data assimilation method, the experiment of $Q_{\text {in }}$ identification was conducted by the real case Red River. In this case, $Q_{\text {in }}$ was considered only control variable and identified with a single serie data at Hanoi gauging station. The first guess was that $Q_{\text {in }}$ is constant, i.e. $Q_{\text {in }}=6000 \mathrm{~m}^{3} / \mathrm{s}$. Other parameters, such as initial conditions, outflow boundary condition and manning coefficients, were given. The stop criterion $\varepsilon$ of the convergence of minimization was set at $10^{-4}$. The maximum number of iteration steps was limited to 50 for contrasting.

\subsection{Water levels boundary}

The water levels at downstream are quiet reliable. Tab. 3 shows water levels carried out by both Hanoi Hydrology Station and National Hydro meteorological Center.

Region is divided into computational grid 19101 nodes and 36199 elements. Simulation time to shorten to 24000 seconds, the time step is 0.5 seconds, a total of 48.000 time step. Point hydrological measurement stations Hanoi, in element number 1075, UTM coordinates $(512084 ; 2325614)$.

Region also contains many alluvial plains so our code must be able to treat the wet/dry problems. 




Fig. 3. Study area and data

Table 3. Water levels

\begin{tabular}{|l|c|}
\hline & Hanoi Station and NCHMF (m) \\
\hline Son Tay & $9.72 \mathrm{~m}$ \\
\hline Thuong Cat & $5.63 \mathrm{~m}$ \\
\hline Hanoi & $6.36 \mathrm{~m}$ \\
\hline Hung Yen & $3.66 \mathrm{~m}$ \\
\hline
\end{tabular}




\section{MATHEMATICAL MODEL}

\subsection{Shallow water equations}

The two dimensional form of the Shallow Water Equation (SWE) with variable topography and taking into account bed shear stress in conservative form

$$
\begin{aligned}
& \partial_{t} \mathbf{h}+\operatorname{div}(\mathbf{q})=0, \\
& \partial_{t} \mathbf{q}+\operatorname{div}\left(\frac{1}{\mathbf{h}} \mathbf{q} \otimes \mathbf{q}\right)+\frac{1}{2} \mathbf{g} \nabla \mathbf{h}^{2}+\mathbf{g h} \nabla z_{b}+\mathbf{g} \frac{\mathbf{n}_{b}{ }^{2}\|\mathbf{q}\|_{2}}{\mathbf{h}^{7 / 3}} \mathbf{q}=\mathbf{0},
\end{aligned}
$$

where $h$ is water depth, $\mathbf{q}=(h u, h v)^{T}$-the depth-average velocity vector, $g$-the gravity acceleration, $z_{b}$-the bottom elevation (topography/bathymetry), and $n_{b}\left[\mathrm{~m}^{1 / 3} \sqrt{\mathrm{s}}\right]$-the Manning coefficient for the bed roughness. The initial conditions are

$$
h(0)=h_{0}, \quad \mathbf{q}(0)=\mathbf{q}_{0} .
$$

The model is closed with appropriate boundary conditions depending on the considered case (prescribed inflow, free outflow, fixed-depth outflow, etc.)

$$
\begin{array}{lll}
(\overrightarrow{\mathbf{q}} \cdot \overrightarrow{\mathbf{n}})=-\mathbf{q}_{\text {in }} & \text { on } & \Gamma_{\text {in }} \\
(\overrightarrow{\mathbf{q}} \cdot \overrightarrow{\mathbf{n}})=0 & \text { on } & \Gamma_{\text {wall }} \\
\mathbf{q}_{\text {out }}=\alpha\left(\mathbf{h}-z_{\text {Ref }}\right)^{\beta} & \text { on } & \Gamma_{\text {out }} \\
\partial_{\mathbf{n}}(\mathbf{u} \cdot \mathbf{n}+2 c)=0 & \text { on } & \Gamma_{\text {out }}
\end{array}
$$

where $\mathbf{q}=h \mathbf{u}$ is the unit discharge, $h_{0}$ is the initial water depth-field and $q_{0}$ is the initial unit discharge field, $\mathbf{n}$ is outward normal to the boundary.

\subsection{Adjoint system}

Considering a cost function of the form

$$
\mathbf{J}(\mathbf{k})=\int_{0}^{T}\left(\left\|\mathbf{h}(t)-\mathbf{h}_{\mathrm{obs}}(t)\right\|_{\Omega}^{2}+\left\|\mathbf{q}(t)-\mathbf{q}_{\mathrm{obs}}(t)\right\|_{\Omega}^{2}\right) \mathrm{d} t .
$$

Variational data assimilation relies on minimizing cost function $\mathbf{J}$ using the socalled adjoint model. The adjoint method makes it possible to compute efficiently all partial derivatives of the cost function $\mathbf{J}$ with respect to the components of the control vector $\mathbf{k}$. The adjoint model for states and adjoint variables in the system (1), (2), (3) is obtained as follows

$$
\begin{aligned}
& \partial_{t} \tilde{h}-\mathbf{u} \cdot(\mathbf{u} \cdot \nabla) \tilde{\mathbf{q}}+g h \operatorname{div}(\tilde{\mathbf{q}})-g \tilde{\mathbf{q}} \cdot \nabla z_{b}+\frac{7}{3} g \frac{n^{2}\|\mathbf{q}\|}{h^{4 / 3}} \mathbf{u} \tilde{\mathbf{q}}=\left(h(t)-h_{\mathrm{obs}}(t)\right), \\
& \partial_{t} \tilde{\mathbf{q}}+\nabla \tilde{h}+(\mathbf{u} \cdot \nabla) \tilde{\mathbf{q}}+(\nabla \tilde{\mathbf{q}})^{T} \mathbf{u}-g \frac{n_{b}{ }^{2}\|\mathbf{u}\|}{h^{4 / 3}} \tilde{\mathbf{q}}-g \frac{n_{b}{ }^{2}}{h^{4 / 3}\|\mathbf{u}\|}(\mathbf{u} \otimes \mathbf{u}) \tilde{\mathbf{q}}=0, \\
& \tilde{h}(T)=0, \quad \tilde{\mathbf{q}}(T)=0, \\
& \tilde{\mathbf{q}}=0 \quad \text { on } \Gamma_{\text {in }} \\
& (\tilde{\mathbf{q}} \cdot \mathbf{n})=0 \quad \text { on } \Gamma_{\text {wall }} \\
& (\tilde{\mathbf{h}}+2(\mathbf{u} . \mathbf{n})(\tilde{\mathbf{q}} \cdot \mathbf{n}))=0 \text { on } \Gamma_{\text {out }}
\end{aligned}
$$


The integrating backward in time the adjoint gives a solution. Then the partial derivatives of the cost function are simple functions of the state variables. We have

$$
\begin{aligned}
& \frac{\partial J}{\partial h_{0}}(\mathbf{k})=-\tilde{h}(0), \\
& \frac{\partial J}{\partial \mathbf{q}_{0}}(\mathbf{k})=-\tilde{\mathbf{q}}(0), \\
& \frac{\partial J}{\partial z_{b}}(\mathbf{k})=-\int_{0}^{T} \operatorname{div}(g h(t) \tilde{\mathbf{q}}(t)) \mathrm{d} t, \\
& \frac{\partial J}{\partial z_{b}}(\mathbf{k})=2 g n \int_{0}^{T}\|\mathbf{u}(t)\| h(t)^{-\frac{1}{3}} \mathbf{u}(t) \tilde{\mathbf{q}}(t) \mathrm{d} t, \\
& \frac{\partial J}{\partial \mathbf{q}_{\text {in }}}(\mathbf{k})=-\tilde{h} .
\end{aligned}
$$

The control variables are the initial conditions $h_{0}$ and $q_{0}$, Manning coefficient $n$, topography of the bottom $z_{b}$ and flow rate upstream $q_{\text {in }}$. We have so the complete control vector $\mathbf{k}=\left(h_{0}, q_{0}, n, z_{b}, \mathbf{q}_{\text {in }}\right)$.

These partial derivatives are used as inputs for the minimization algorithm. A simple integration of the direct model followed by a single backward integration in time of the adjoint model is sufficient to calculate all the components of the gradient of the cost function. Minimization of the cost function is performed in practice through a type algorithm Quasi-Newton (BFGS): the optimization M2QN1 module developed by Gilbert and Lemarechal [9].

\section{SENSITIVITY ANALYSIS OF PARAMETERS}

For data assimilation method, the input parameters include initial conditions, bottom topography, flow rate and water level at open boundary, Manning roughness coefficient. The input parameters of the real problem always contain errors so they are sensitive to the model results. The evaluation of the reliability and comparative the sensibility for each parameter to model are needed.

Table 4. The sensibility to parameter

\begin{tabular}{|l|c|c|}
\hline Input parameters & Value & Sensibility \\
\hline Bottom topography & & $99.97 \%$ \\
\hline Inflow flow rate & $6000 \mathrm{~m}^{3} / \mathrm{s}$ & $3.5186 \mathrm{E}-003 \%$ \\
\hline Outflow water level & $5.63 \mathrm{~m}$ & $1.2545 \mathrm{E}-004 \%$ \\
& $3.66 \mathrm{~m}$ & \\
\hline Manning coefficient & 0.025 & $1.618 \mathrm{E}-002 \%$ \\
\hline Initial condition $h$ & & $9.43 \mathrm{E}-003 \%$ \\
\hline Initial condition $u$ & & $7.6259 \mathrm{E}-006 \%$ \\
\hline Initial condition $v$ & & $5.8954 \mathrm{E}-006 \%$ \\
\hline
\end{tabular}


The first question posed is that when there is a small change of the input parameters how the results will be changed. Mathematically speaking, this method calculates the partial derivatives of the objective function of the input parameters.

Parameters of the upstream flow rate are generally affected by many factors so it is not accurate. Some authors use the ratting curves, but this relationship lines are never stable, changed after each flood season. The measurements by ADCP are very rare and expensive, and also dependent on factors of speed, flow rate, etc.

From Tab. 4, we find that the parameter bottom topography is the most sensitive. Then the Manning coefficient is the second, initial condition, flow rate, and water level are second, third, fourth and last ones, respectively. It means that a small change of the bottom topography will lead to big changes of results.

\section{IDENTIFICATION OF INPUT PARAMETER}

Actually, we can not identify all parameters at once because our program is still developing and we miss a lot of field data, power computing devices with very large memory capacity. The calculation of data assimilation methods has to save all the state variables at each time step. The long computation time on large areas will need powerful computing devices and very large memory capacity. Our computer is 128GRAM, with MPI of Centre for Informatics and Computing, Vietnam Academy of Science and Technology. So we try to identify only some parameters assuming other parameters are known, although the job is not easy.

\section{Identification of flow rate}

We suppose that we know all parameters except the inflow flow rate. The coefficient Manning is 0.025. With another coefficient Manning is 0.02 we have also tested.

We see that in both case about 4000 first seconds, the flow rate curve is very oscillation. Because of we have input a bad initial condition. From 4000 first seconds, the flow rate curve is stable and converges to the correct value of inflow flow rate (see Fig. 4).

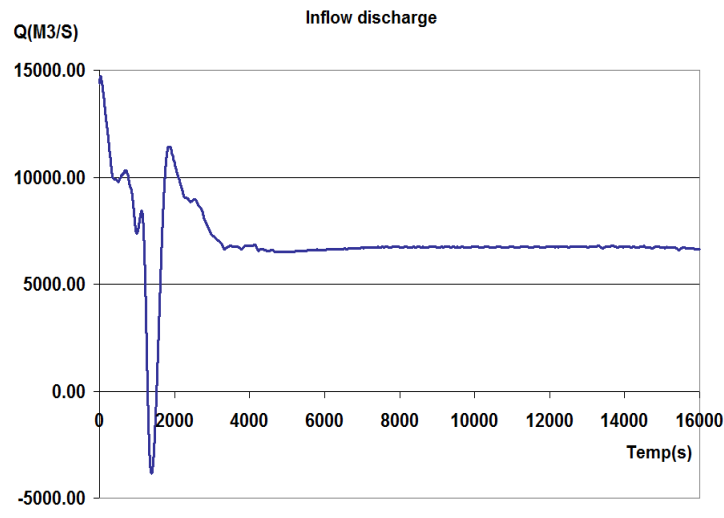

(a)

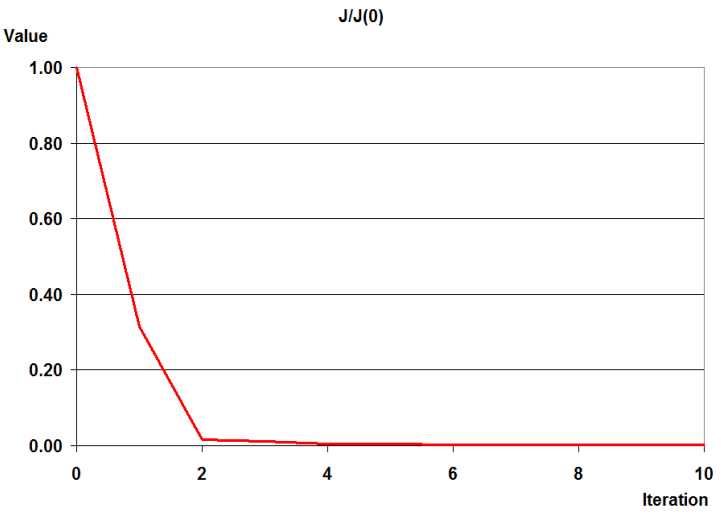

(b)

Fig. 4. a) Flow rate, b) Cost function 


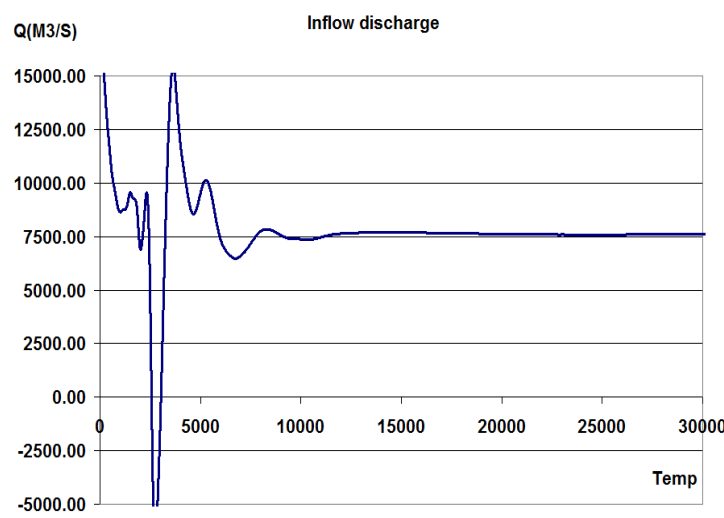

(a)

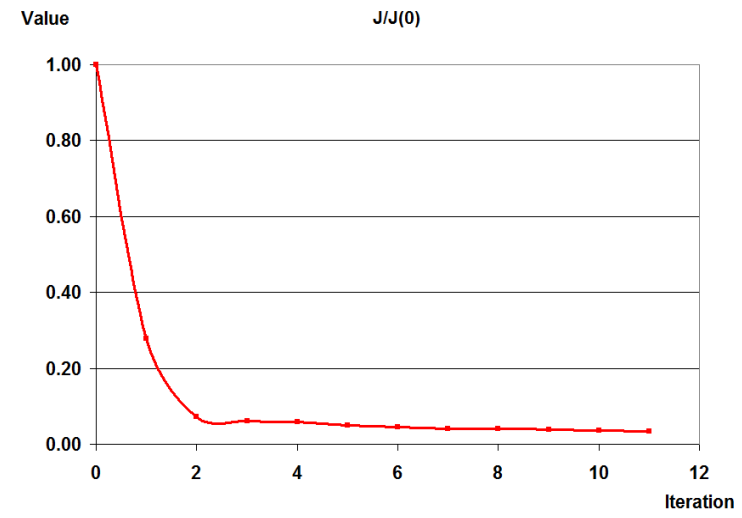

(b)

Fig. 5. a) Flow rate, b) Cost function

The following Tab. 5 shows the comparison between the results of many authors.

Table 5. The results of different method

\begin{tabular}{|l|c|c|}
\hline & $\begin{array}{c}\text { Discharge Son Tay } \\
\text { Station }\left(\mathrm{m}^{3} / \mathrm{s}\right)\end{array}$ & $\begin{array}{c}\text { Percentage } \\
\text { errors }\end{array}$ \\
\hline Discharge Ratings of Hanoi Station & $11000 \mathrm{~m}^{3} / \mathrm{s}$ & $57 \%$ \\
\hline Dischrge Ratings of NCHMF & $8885 \mathrm{~m}^{3} / \mathrm{s}$ & $26 \%$ \\
\hline ADCP & $8022 \mathrm{~m}^{3} / \mathrm{s}$ & $14,6 \%$ \\
\hline DA Manning 0.025 & $6730 \mathrm{~m}^{3} / \mathrm{s}$ & $3,8 \%$ \\
\hline DA Manning 0.02 & $7590 \mathrm{~m}^{3} / \mathrm{s}$ & $8,4 \%$ \\
\hline Official data & $7000 \mathrm{~m}^{3} / \mathrm{s}$ & \\
\hline
\end{tabular}

From the total inflow discharge flow into system Red River from two lakes Hoa Binh, Tuyen Quang and Yen Bai that day (Fig. 5), we found that our result has less percentage errors, so is more justifiable and reasonable than that of other authors.

\section{OVERLAPPING STRATEGY AND IMPROVEMENT OF COMPUTATION COST}

The adjoint method makes it possible to efficiently compute the gradients of J with respect to control variables. However, the computational cost, including the CPU time and memory requirement, is much higher than that of the classical forward model. The memory requirement, in particular, is enormous. Basically, one needs to store all the state variables $U$ from the initial time to the final time at each control volume, in order to solve the adjoint model backward in time. Nevertheless, this is not completely true since automatic differentiation include a strategy for balancing storage and recomputation, but the storage approach still remains the basis for adjoint model computation. Thus, the typical 
amount of memory is insufficient for some practical long-duration flood modeling. B. Luong, J. Blum, and J. Verron, [10]; propose a temporal strategy that consists of splitting the whole assimilation period into several sub-intervals, then carrying out the assimilation process sequentially over each sub-interval. The final states of the previous sub-interval serve as the first guess of the subsequent sub-interval. They focused on the accuracy of the final time solution for a quasi-geostrophic oceanographic model (the control variable is the initial condition). Then, they defined a "progressive" temporal strategy that better identified for the initial control state.

In the study of J. Monier et al., [7], they adapted the sequential temporal strategy of B. Luong, J. Blum, and J. Verron [10] to the specific components of their river hydraulics problem: the identification of inflow hydrograph (time-dependent parameter), Manning coefficients and/or initial conditions (time-independent parameters). In the following descriptions of the assimilation experiments, they refered the previous sequential strategy as the "temporal strategy without overlapping" and the new strategy defined as the "temporal strategy with overlapping".

\subsection{Temporal strategy without overlapping}

The temporal strategy without overlapping efficiently identifies time-independent parameters: from the observations listed above, we identified the Manning roughness coefficients in three different land-use areas (the main channel, left floodplain and right floodplain).

The first guess of one sub-interval was the converged value of the previous subinterval. The mean values of the resulting Manning coefficients are close to the true values. The temporal strategy without overlapping works well for the identification of Manning coefficients which are time-independent parameters.

Since there is no overlapping in time and since the speed of convergence of the minimization algorithm is not affected by the time-splitting, the time computation of the identification process is similar to that of the process without the temporal strategy. But, the required memory is roughly divided by the number of sub-intervals, much less than that of the original process (see [7]).

\subsection{Temporal strategy with overlapping}

They adapted the sequential temporal strategy of B. Luong, J. Blum, and J. Verron [10] (i.e. the temporal strategy without overlapping) to the specific river hydraulics problems. As a matter of fact, the strategy must be adapted to time-dependent parameters like inflow discharge, since information takes time to propagate to the location of observations.

Hence, if observations are not dense enough there is a blind period during which the time-dependent parameter is not identifiable. This phenomenon is apparent at the end of the period of assimilation in all of the previously presented figures: the inflow is not well simulated, but rather extended because of the regularization term. In fact, during this period of time, inflow values have no effect on the observations. The blind period represents the time of response of the control variables to the observations. Therefore, we cannot apply the temporal strategy without overlapping to simulate inflow. They propose a new temporal strategy with overlapping. The assimilation period is divided into 
several sub-intervals as it was in previous experiments, but with overlapping. They denote the length of this overlapped period with Tretro (retrogressive time). The initial conditions for the new subsequence are defined by the computed flow state of the previous sub-interval. The first guess of control variables in the new sub-sequence is defined as the value identified during the previous sub-interval (see J. Monier et al., [7]).

\section{CONCLUSIONS}

Various aspects related to the sensitivity and data assimilation for flood modeling were discussed in this paper. The computational software Dassflow-Shallow was developed to provide a data assimilation framework for modeling shallow water flows. Its assimilation method (4D-var) relies on the estimation of the gradients of the cost function using adjoint code and allows sensitivity analysis to several parameters and data. Thereafter the sensitivity analysis was also conducted on the real case with complex topography to assess the potential of the method for operational flood modeling.

The sensitivity analysis to all parameters for the real case showed that the variational method allowed us to plot sensitivity maps taking into account observations from stations. We thus were able to locate zones particularly sensitive to parameters and to quantify their importance. The automatic parameter recovering showed good results for uniform flow rate using a single observation point. Comparing two common actual methods, we found that our result has less percentage errors, so it is more justifiable and reasonable than that of other authors.

This work is still in progress. Several future developments are planned, for instance, temporal sensitivity analysis to highlight zones of influence around the peak flow. Researches in numerical schemes and optimization are also being conducted. Twin experiment for the real case is still facing technical issues (adjoint code parallelization, memory overflows) and should be addressed in a future paper. Finally the assessing the method for real-time or operational forecasting is expected, particularly by Ministry of Water in Vietnam.

\section{ACKNOWLEDGMENTS}

This work is part of the VAST project funded.

\section{REFERENCES}

[1] World Meteorological Organization. Manual on stream gauging, (2003).

[2] L. Bertino. Assimilation de données pour la prédiction de paramètres hydrodynamiques et écologiques: cas de la lagune de l'Oder. PhD thesis, École Nationale Supérieure des Mines de Paris, (2001).

[3] H. Roux and D. Dartus. Estimating hydraulic parameters and geometric characteristics of a river from remote sensing data using optimization methods. In Second International conference on Fluvial Hydraulics, Naples, Italy, (2004), pp. 1443-1451.

[4] W. Castaings. Analyse de sensibilité et estimation de paramètres pour la modélisation hydrologique: potentiel et limitations des méthodes variationnelles. PhD thesis, Université Joseph-FourierGrenoble I, (2007). 
[5] M. Honnorat, X. Lai, J. Monnier, and F.-X. Le Dimet. Variational data assimilation for 2D fluvial hydraulics simulations. In CMWR XVI-Computational Methods for Water Ressources. Copenhagen, (2006), pp. 1-8.

[6] M. Honnorat, J. Marin, J. Monnier, and X. Lai. Dassflow v1.0: A variational data assimilation software for 2D river flows, (2007).

[7] X. Lai and J. Monnier. Assimilation of spatially distributed water levels into a shallow-water flood model. Part I: Mathematical method and test case. Journal of Hydrology, 377, (1), (2009), pp. 1-11.

[8] N. T. Don, N. V. Que, N. Q. Minh, N. H. Phong, and T. Q. Hung. Applying data assimilation method in Red river system. In 19th IAHR-APD Congress, (2014), pp. 207-211.

[9] J. C. Gilbert and C. Lemaréchal. Some numerical experiments with variable-storage quasinewton algorithms. Mathematical programming, 45, (1-3), (1989), pp. 407-435.

[10] B. Luong, J. Blum, and J. Verron. A variational method for the resolution of a data assimilation problem in oceanography. Inverse Problems, 14, (4), (1998), pp. 979-997. 